\title{
The quality of dying and death for patients in intensive care units: a single center pilot study
}

\author{
Yanghwan Choi', Myoungrin Park' ${ }^{2}$ Da Hyun Kang ${ }^{3}$, Jooseon Lee ${ }^{4}$ Jae Young Moon ${ }^{3}$, Heejoon Ahn ${ }^{5}$ \\ ${ }^{1}$ Department of Burn and Critical Care, Bestian Hospital, Osong; ${ }^{2}$ Department of Internal Medicine, Daejeon Veterans Hospital, Daejeon; ${ }^{3}$ Division of \\ Pulmonary and Critical Care Medicine, Department of Internal Medicine and ${ }^{4}$ Hospital Ethics Committee, Chungnam National University Hospital, \\ Daejeon; ${ }^{5}$ Department of Internal Medicine, Gangneung Asan Hospital, University of Ulsan College of Medicine, Gangneung, Korea
}

Background: To identify the necessary care for dying patients in intensive care units (ICUs), we designed a retrospective study to evaluate the quality of dying and death (OODD) experienced by the surrogates of patients with medical illness who died in the ICU of a tertiary referral hospital.

Methods: To achieve our objective, the authors compared the QODD scores as appraised by the relatives of patients who died of cancer under hospice care with those who died in the ICU. For this study, a Korean version of the QODD questionnaire was developed, and individual interviews were also conducted.

Results: Sixteen people from the intensive care group and 23 people from the hospice care group participated in the survey and completed the questionnaire. The family members of patients who died in the ICU declined participation at a high rate (50\%), with the primary reason being to avoid bringing back painful memories (14 people, 87.5\%). The relatives of the intensive care group obtained an average total score on the 17-item OODD questionnaire, which was significantly lower than that of the relatives of the hospice group (48.7 $\pm 15.5 \mathrm{vs}$. $60.3 \pm 14.8, P=0.03)$.

Conclusions: This work implies that there are unmet needs for the care of dying patients and for the QODD in tertiary hospital ICUs. This result suggests that shared decision making for advance care planning should be encouraged and that education on caring for dying patients should be provided to healthcare professionals to improve the QODD in Korean ICUs.

Key Words: advance care planning; education; end-of-life care; intensive care unit; quality of life; surveys and questionnaires

\section{INTRODUCTION}

More than $75 \%$ of all deaths in Korea occur in hospitals [1]. However, domestic studies on the quality of dying and death in hospitals are rare. In intensive care units (ICUs), a large number of patients with terminal illnesses die while receiving life-sustaining treatments. Treating terminally ill patients with dignity is an important role for medical professionals [2]. For this reason, it is necessary to survey the quality of dying and death for patients in ICUs and hospices.

The quality of dying and death (QODD) is a tool proposed by Patrick et al. [3] through systematic literature studies that measures the quality of care for dying patients. The tool's validity and reliability have been evaluated and demonstrated [4], and has been translated into

\section{Original Article}

Received: November 26, 2018

Revised: April 16, 2019

Accepted: May 3, 2019

\section{Corresponding author}

Jae Young Moon

Division of Pulmonary and Critical

Care Medicine, Department of

Internal Medicine, Chungnam

National University Hospital,

Chungnam National University

College of Medicine, 282 Munhwa-

ro, Jung-gu, Daejeon 35015, Korea

Tel: +82-42-280-7870

Fax: +82-42-280-7877

E-mail: jymoon@cnuh.co.kr

Copyright (๑ 2019 The Korean Society of Critical Care Medicine

This is an Open Access article distributed under the terms of Creative Attributions Non-Commercial License (http:// creativecommons.org/li-censes/by-nc/4.0/) which permits unrestricted noncommercial use, distribution, and reproduction in any medium, provided the original work is properly cited. 
other languages, including German, Spanish, and Korean [5-7].

To identify the necessary care for dying patients in ICUs, we studied the quality of dying and death experienced by the surrogates of patients with medical illnesses who died in the ICU of a tertiary referral hospital. For this, the authors compared the QODD as appraised by the family members of patients who died of cancer under hospice care with those who died in the ICU. For this study, a Korean version of the QODD questionnaire was developed and individual interviews were conducted.

\section{MATERIALS AND METHODS}

\section{Screening Participants}

The researchers screened medically ill patients over 18 years old who died either in the adult ICU or under hospice care at a single tertiary medical center in the period between October 2016 and October 2017. We reviewed their hospital records for any suggestion of the patient's medical futility, which refers to medically irreversible conditions or an active dying process with or without life-sustaining treatment. Based on the medical records, major medical decision makers or family members were selected for the questionnaire survey. Patients whose deaths occurred within the previous 4 weeks were excluded from the study. Also excluded were those deceased who had stayed for less than 72 hours at the ICU and who received hospice care for 2 weeks or less. Relatives who declined to participate in the questionnaire survey were excluded from the comparative analysis. Their reasons for nonparticipation were noted.

\section{Study Design and Ethical Considerations}

The institute in which this study was carried out is a tertiary referral medical center. The ICUs in this center have 56 beds and the hospice has 15 beds. The hospice at the hospital is the only accredited facility in the district that provides in-hospital care, home-visiting care, and consultative services. The hospice employs trained specialists. The institute provided standard pain management for the care of dying patients according to guidelines $[2,8,9]$.

Patient information was collected from medical records. A standardized questionnaire for the QODD was used during individual interviews conducted by trained researchers. When face-to-face interviews were not available, postal or email correspondences were used. Two researchers, one from the ICU and the other from the hospice, underwent two training sessions on the questionnaire to obtain correct responses and eliminate potential biases.

\section{KEY MESSAGES}

- The survey participation rate from the relatives of the intensive care group was lower $(50.0 \%)$ than that of the hospice group (76.5\%), with the reason of avoiding painful memories.

- The total score of the quality of dying and death (QODD) from the family members of the intensive care group was statistically significantly lower than that of the hospice group.

- This study implies that there are unmet needs for the care of dying patients and for the quality of dying and death in intensive care units.

The purpose of the study was explained to the survey participants. The participants consented to the study by participating in the survey, and the consent form was waived. This study was approved by the Ethics Committee of Chungnam National University Hospital (IRB No. 2018-03-038-001).

\section{Questionnaire of the Quality of Dying and Death}

This study adopted the "Quality of Dying and Death-17-item version" developed by Downey et al. [10], which employed 17 questions of the highest priority from the original "Quality of Dying and Death-31-item version" [4]. The questions were translated into Korean before they were revised and finalized after review by four researchers. Each question has two parts. The first asks relatives how often the deceased experienced each item using a scale, where 0 is "none of the time" and 5 is "all of the time." Some questions require a "yes" or "no" response instead of a scale. The second part asks relatives to rate that aspect of the deceased's dying experience on a scale from 0 to 10 , where 0 is a "terrible experience" and 10 is an "almost perfect experience." A total score was presented within a range of 0 to 100 after adding the sum of all the scores from the questionnaire and dividing it by the number of questions. This means that the higher the total score, the greater the quality of dying and death [4]. The 17-item questionnaire consists of the following five domains: (1) symptoms and personal control; (2) preparation for death; (3) family; (4) treatment preferences; and (5) whole person concerns [10].

\section{Statistical Analysis}

Data were presented as the mean or median for continuous variables or as numbers with percentages for categorical variables. Non-normally distributed data were expressed as ranges with minimum and maximum value. We used skewness to measure the asymmetry of the probability distribution. For a 
unimodal distribution, negative skewness indicates that the tail on the left side of the probability density function is longer than on the right. Conversely, positive skewness indicates that the tail on the right side is longer than on the left.

Nominal variables were compared using a chi-square or Fisher exact test, and the mean values in the two groups were compared using a Student t-test. Non-normally distributed continuous variables were compared using a Mann-Whitney U-test. Statistical significance was defined as $\mathrm{P}<0.05$. Statistical analyses were performed using PASW ver. 18.0 (SPSS Inc., Chicago, IL, USA).

\section{RESULTS}

\section{Participants}

During the study period, 1,289 medically ill patients were admitted into the ICU. Among them, 92 patients died while receiving medical treatments in the ICU. The researchers screened 40 patients eligible for the selection criteria.

The surrogates of eight patients were not reachable. Of the remaining 32, 16 surrogates participated in the face-to-face survey and completed the questionnaire (participation rate of $50 \%$; completion rate of $100 \%$ ) (Figure 1). The main reason given for declining participation was to avoid bringing back painful memories (14 people, $87.5 \%$ ).

During the study period, 93 terminally ill cancer patients died while on in-hospital hospice care or home-visiting hospice care. Among them, 34 relatives were eligible for the selection criteria. Twenty-six surrogates agreed to participate in the survey, but only 23 of them completed the questionnaire, either by 16 postal correspondences or seven face-to-face interviews (participation rate, $76.5 \%$; completion rate, $88.5 \%$ ) (Figure 1).

The median age of the participants in the intensive care group was 52 years. The majority of the survey participants from this group were adult children (15 participants, 93.8\%) (Table 1). The median age of the participants in the hospice group was 55 years, and among these participants, 12 adult children (56.5\%) and nine spouses (39.1\%) participated in the survey.

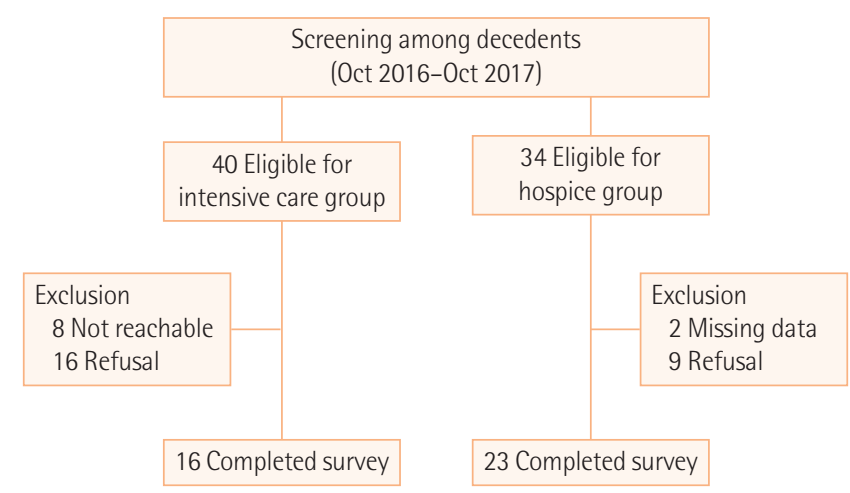

Figure 1. Flowchart of the survey.

Table 1. Demographics of the decedents and respondents who participated in a QODD questionnaire

\begin{tabular}{|c|c|c|c|c|}
\hline \multirow{2}{*}{ Variable } & \multicolumn{2}{|c|}{ Intensive care group $(n=16)$} & \multicolumn{2}{|c|}{ Hospice group ( $n=23$ ) } \\
\hline & Decedent & Respondent & Decedent & Respondent \\
\hline Age, median (range) & $82(69-87)$ & $52(32-75)$ & $68(54-90)$ & $55(29-76)$ \\
\hline Male sex & 12 & 0 & 15 & 9 \\
\hline \multicolumn{5}{|l|}{ Education } \\
\hline$\leq 6$ th grade & 6 & 0 & 7 & 1 \\
\hline$\leq 7$ th-9th grade & 4 & 3 & 4 & 2 \\
\hline$\leq 10$ th-12th grade & 2 & 4 & 8 & 8 \\
\hline College & 4 & 5 & 2 & 9 \\
\hline Post-college & 0 & 4 & 2 & 3 \\
\hline \multicolumn{5}{|l|}{ Insurance } \\
\hline Medicare & 14 & - & 20 & - \\
\hline Medicaid & 2 & - & 3 & - \\
\hline \multicolumn{5}{|l|}{ Relationship to decedent } \\
\hline Spouse & - & 1 & - & 9 \\
\hline Adult child & - & 15 & - & 13 \\
\hline Other family member & - & - & - & 1 \\
\hline
\end{tabular}

OODD: quality of dying and death. 


\section{Characteristics of Patients}

The median age of the deceased from the intensive care group was 82 years, and 12 patients from this group were male $(75.0 \%)$ (Table 2). In this group, infection-related illnesses accounted for $56.2 \%$ of deaths, including six deaths from pneumonia and three deaths caused by other infections (Table 2). The median length of stay in the ICU was 18 days. Fourteen patients died in the ICU, while two patients died in a general ward after being transferred for end-of-life care in their last days. All 16 patients received active intensive care for prolonging their life, such as mechanical ventilation, within 7 days of their death, but their conditions were medically irreversible (Supplementary Table 1$)$. They had all provided a documented $(81.2 \%)$ or verbal (18.8\%) DNR (do not resuscitate) order, according to advance directive (6.3\%) or advance care planning (93.7\%) with their surrogate.

The median age of the 23 patients from the hospice group was 68 years. Fifteen of these were male (65\%) (Table 2). The

Table 2. Demographics of intensive care group and hospice care group

\begin{tabular}{|c|c|c|}
\hline Variable & $\begin{array}{l}\text { Intensive care } \\
\text { group }(n=16)\end{array}$ & $\begin{array}{l}\text { Hospice group } \\
\quad(n=23)\end{array}$ \\
\hline Age, median (range) & $82(69-87)$ & $68(54-90)$ \\
\hline Male sex, \% (n) & $75(12)$ & $65(15)$ \\
\hline \multicolumn{3}{|l|}{ Causes of death } \\
\hline Solid cancer & 2 & 22 \\
\hline Hematologic malignancy & 2 & 1 \\
\hline Cardiovascular diseases & 2 & - \\
\hline \multicolumn{3}{|l|}{ Sepsis } \\
\hline Pneumonia & 6 & - \\
\hline Other origin infection & 3 & - \\
\hline Cerebrovascular disease & 1 & - \\
\hline \multicolumn{3}{|l|}{ Place of death } \\
\hline Intensive care unit & 14 & - \\
\hline General ward-deathbed & 2 & - \\
\hline Hospice ward/facility & - & 10 \\
\hline Home & - & 13 \\
\hline Length of ICU stay, median (range) & $17.5(5-46)$ & - \\
\hline APACHE II score & 30 & - \\
\hline \multicolumn{3}{|c|}{ Mechanical support during the last 7 days } \\
\hline Mechanical ventilation & 16 & - \\
\hline Hemodialysis & 8 & - \\
\hline ECMO & 2 & - \\
\hline
\end{tabular}

ICU: intensive care unit; APACHE: Acute Physiologic Assessment and Chronic Health Evaluation; ECMO: extracorporeal membrane oxygenation. causes of all of the deaths in this group were cancers (22 solid cancers and one hematologic malignancy). Ten patients died in the hospice, while 13 patients died in their homes. None of the patients received any life-sustaining treatments such as mechanical ventilation during the last 7 days prior to their deaths.

\section{The Quality of Death}

QODD total score

The average total score on the 17-item QODD questionnaire from the family members of the intensive care group was 48.7 (standard deviation [SD], 15.5; skewness value, 0.114). The average total score from the family members of the hospice group was 60.3 (SD, 14.8; skewness value, 0.164 ). The lower average QODD total score from the intensive care group was statistically significant $(\mathrm{P}=0.03)$ (Figure 2$)$.

\section{QODD by domains and items}

\section{Symptoms and personal control}

Although the patients in the intensive care group had been provided pain management according to pain assessment protocols, their relatives' rating for the QODD "pain under control" item was lower than that of the hospice group (2.1 vs. $5.3, \mathrm{P}=0.001$ ) (Table 3 ). The intensive care group relatives' rating for the "breathing comfort" item was significantly lower than that of the hospice group (2.1 vs. 4.1, $\mathrm{P}=0.032$ ) (Table 3).

\section{Preparation for death}

The relatives of the intensive care group provided ratings for the "untroubled about strain on loved ones" item and the "fu-

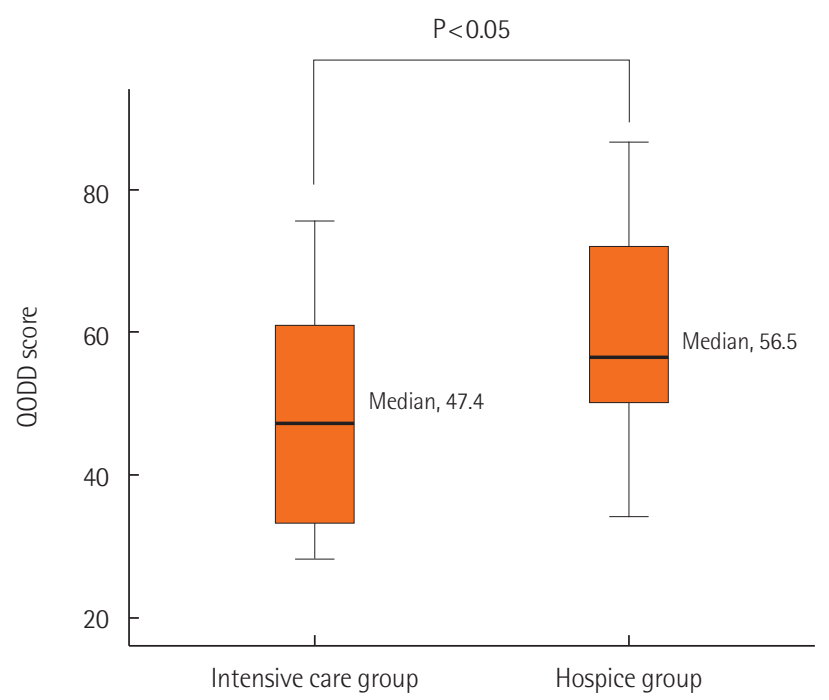

Figure 2. Comparison of quality of dying and death (QODD) between the intensive care group and hospice care group. 
Table 3. Comparison of family respondents' ratings between the intensive care group and hospice care group

\begin{tabular}{|c|c|c|c|c|c|}
\hline \multirow{2}{*}{ Item } & \multicolumn{2}{|c|}{ Intensive care group $(n=16)$} & \multicolumn{2}{|c|}{ Hospice group $(n=23)$} & \multirow{2}{*}{ P-value } \\
\hline & Value & Skew & Value & Skew & \\
\hline \multicolumn{6}{|l|}{ Symptom and personal control } \\
\hline \multicolumn{6}{|l|}{ Pain under control } \\
\hline Decedent's experience & $3.8 \pm 1.4$ & -1.54 & $3.2 \pm 1.1$ & 0.16 & 0.079 \\
\hline Respondents' rating & $2.1 \pm 2.1$ & 1.44 & $5.3 \pm 3.1$ & 0.10 & 0.001 \\
\hline \multicolumn{6}{|c|}{ Control over what was going on } \\
\hline Decedent's experience & $1.3 \pm 2.1$ & 1.23 & $1.7 \pm 1.5$ & 0.48 & 0.228 \\
\hline Respondents' rating & $2.4 \pm 2.8$ & 1.39 & $3.8 \pm 2.7$ & 0.38 & 0.114 \\
\hline \multicolumn{6}{|l|}{ Control of bladder and bowels } \\
\hline Decedent's experience & $3.1 \pm 2.2$ & -0.77 & $1.8 \pm 1.9$ & 0.46 & 0.074 \\
\hline Respondents' rating & $5.5 \pm 4.4$ & -0.25 & $4.2 \pm 3.1$ & 0.13 & 0.288 \\
\hline \multicolumn{6}{|l|}{ Breathing comfort } \\
\hline Decedent's experience & $1.9 \pm 1.8$ & 0.36 & $3.0 \pm 1.4$ & -0.53 & 0.065 \\
\hline Respondents' rating & $2.1 \pm 2.7$ & 1.86 & $4.1 \pm 3.0$ & 0.10 & 0.032 \\
\hline \multicolumn{6}{|l|}{ Preparation for death } \\
\hline \multicolumn{6}{|l|}{ At peace with dying } \\
\hline Decedent's experience & $3.0 \pm 2.2$ & -0.36 & $3.3 \pm 1.7$ & -0.91 & $>0.999$ \\
\hline Respondents' rating & $3.4 \pm 4.0$ & 0.82 & $5.8 \pm 3.5$ & -0.35 & 0.057 \\
\hline \multicolumn{6}{|l|}{ Unafraid of dying } \\
\hline Decedent's experience & $2.9 \pm 1.8$ & -0.12 & $3.0 \pm 1.8$ & -0.60 & 0.899 \\
\hline Respondents' rating & $3.3 \pm 3.4$ & 1.16 & $5.0 \pm 2.9$ & -0.33 & 0.107 \\
\hline \multicolumn{6}{|c|}{ Untroubled about strain on loved ones } \\
\hline Decedent's experience & $2.3 \pm 2.0$ & -0.04 & $2.7 \pm 1.3$ & 0.45 & 0.544 \\
\hline Respondents' rating & $7.4 \pm 3.0$ & -1.15 & $4.8 \pm 2.4$ & 0.10 & 0.007 \\
\hline \multicolumn{6}{|l|}{ Healthcare costs covered } \\
\hline Yes & $81.3(13)$ & - & $87.0(20)$ & - & 0.674 \\
\hline Respondents' ratings & $8.3 \pm 3.5$ & -1.71 & $7.8 \pm 3.3$ & -1.34 & 0.360 \\
\hline \multicolumn{6}{|l|}{ Spiritual advisor visits } \\
\hline Yes & $62.5(10)$ & - & $65.2(15)$ & - & $>0.999$ \\
\hline Respondents' ratings & $8.1 \pm 3.0$ & -1.65 & $7.5 \pm 2.4$ & -1.25 & 0.251 \\
\hline \multicolumn{6}{|l|}{ Funeral arrangements in order } \\
\hline Yes & $31.3(5)$ & & $65.2(15)$ & - & 0.054 \\
\hline Respondents' ratings & $8.1 \pm 3.5$ & -1.34 & $7.1 \pm 2.2$ & -0.55 & 0.043 \\
\hline \multicolumn{6}{|l|}{ Goodbyes said } \\
\hline Yes & $37.5(6)$ & - & $47.8(11)$ & - & 0.743 \\
\hline Respondents' ratings & $3.5 \pm 4.1$ & 0.69 & $6.3 \pm 3.4$ & -0.33 & 0.030 \\
\hline \multicolumn{6}{|l|}{ Family } \\
\hline \multicolumn{6}{|l|}{ Time with spouse/partner } \\
\hline A living spouse or partner & 56.9 (9) & & 78.3 (18) & & \\
\hline Decedent's experience & $3.4 \pm 2.4$ & -0.91 & $4.6 \pm 1.0$ & -2.80 & 0.463 \\
\hline Respondents' ratings & $5.0 \pm 4.1$ & -0.25 & $7.9 \pm 2.3$ & -1.51 & 0.059 \\
\hline
\end{tabular}


Table 3. Continued

\begin{tabular}{|c|c|c|c|c|c|}
\hline \multirow{2}{*}{ Item } & \multicolumn{2}{|c|}{ Intensive care group $(n=16)$} & \multicolumn{2}{|c|}{ Hospice group ( $n=23)$} & \multirow{2}{*}{ P-value } \\
\hline & Value & Skew & Value & Skew & \\
\hline \multicolumn{6}{|l|}{ Time with children } \\
\hline A living children & $93.8(15)$ & & $100.0(23)$ & & \\
\hline Decedent's experience & $3.9 \pm 1.8$ & -1.18 & $3.8 \pm 1.0$ & -0.73 & 0.145 \\
\hline Respondents' ratings & $4.6 \pm 3.5$ & 0.44 & $7.8 \pm 1.7$ & -0.66 & 0.010 \\
\hline \multicolumn{6}{|l|}{ Time with other family/friends } \\
\hline Decedent's experience & $2.6 \pm 2.0$ & -0.08 & $2.7 \pm 1.7$ & -0.07 & 0.877 \\
\hline Respondents' ratings & $6.6 \pm 3.0$ & -1.13 & $6.9 \pm 2.6$ & -0.69 & 0.899 \\
\hline \multicolumn{6}{|l|}{ Treatment preference } \\
\hline \multicolumn{6}{|l|}{ Means to hasten death, if needed } \\
\hline Yes & $50.0(8)$ & - & $100.0(23)$ & - & $<0.001$ \\
\hline Respondents' ratings & $5.2 \pm 3.8$ & 0.09 & $8.2 \pm 1.4$ & 0.14 & 0.053 \\
\hline \multicolumn{6}{|c|}{ Use a MV or a dialysis for life support } \\
\hline Yes & $100.0(16)$ & - & 0 & - & $<0.001$ \\
\hline Respondents' ratings & $5.2 \pm 4.1$ & 0.02 & $8.2 \pm 2.0$ & -1.47 & 0.053 \\
\hline \multicolumn{6}{|l|}{ Whole person concerns } \\
\hline \multicolumn{6}{|l|}{ Ability to laugh and smile } \\
\hline Decedent's experience & $0.9 \pm 1.6$ & 1.54 & $1.7 \pm 1.3$ & 0.68 & 0.024 \\
\hline Respondents' ratings & $3.4 \pm 3.8$ & 0.64 & $4.1 \pm 2.6$ & 0.23 & 0.343 \\
\hline \multicolumn{6}{|l|}{ Physical expressions of affection } \\
\hline Yes & $100.0(16)$ & - & $100.0(23)$ & - & $>0.999$ \\
\hline Respondents' ratings & $6.1 \pm 4.0$ & -0.06 & $8.0 \pm 1.7$ & -0.31 & 0.358 \\
\hline \multicolumn{6}{|c|}{ Maintained dignity and self-respect } \\
\hline Decedent's experience & $1.4 \pm 1.7$ & 0.76 & $4.0 \pm 1.4$ & -1.45 & $<0.001$ \\
\hline Respondents' ratings & $2.6 \pm 3.4$ & 1.29 & $6.5 \pm 3.2$ & -0.61 & 0.001 \\
\hline \multicolumn{6}{|l|}{ General review by respondents } \\
\hline QODD of decedent's last days & $4.3 \pm 4.3$ & 0.40 & $6.9 \pm 2.4$ & -0.99 & 0.126 \\
\hline
\end{tabular}

Values are presented as mean \pm standard deviation or percent (number). Each question has two parts. The first asks relatives how often the deceased experienced each item using a scale, where 0 is "none of the time" and 5 is "all of the time," or using "yes" and "no" for some questions; the latter is presented as a percentage in the table. The second part asks relatives to rate this aspect of the deceased's dying experience on a scale from 0 to 10 , where 0 is a "terrible experience" and 10 is an "almost perfect experience."

MV: mechanical ventilation; OODD: quality of dying and death.

neral arrangements in order" item that were significantly higher than those of the hospice group $(\mathrm{P}=0.007$ and $\mathrm{P}=0.043$, respectively) (Table 3). However, this group's rating for "goodbyes said" was significantly lower than that of the relatives of the hospice group ( 3.5 vs. $6.3, \mathrm{P}=0.030$ ) (Table 3 ).

\section{Family}

On the question, "How often did the deceased spend time with family and friends," the intensive care group's rating for "time with the children" was significantly lower than that of the hospice group ( 4.6 vs. $7.8, \mathrm{P}=0.010$ ).

\section{Treatment preferences}

All patients from the intensive care group had been receiving life-sustaining treatments, including mechanical ventilation. Their relatives' rating for the QODD was significantly lower than that of the hospice group (5.2 vs. 8.2, $\mathrm{P}=0.053$ ).

Whole person concerns

On the question of "dignity and self-respect," the rating of the relatives of the intensive care group was statistically significantly lower than that of the hospice group ( 2.6 vs. $6.5, \mathrm{P}=0.001$ ). 
Table 4. Comparison by subgroups of the decedents in the intensive care unit and factors associated with QODD total score

\begin{tabular}{lcc}
\hline \multirow{2}{*}{\begin{tabular}{l} 
Variable \\
\cline { 2 - 3 }
\end{tabular}} & $\begin{array}{c}\text { Intensive care group }(n=16) \\
(n=8) \text { vs. lower } \\
(n=8) \text { total Q0DD }\end{array}$ & $\begin{array}{c}\text { man's } \\
\text { rho }\end{array}$ \\
\hline $\begin{array}{l}\text { Preparation for death } \\
\text { Untroubled about strain on loved ones }\end{array}$ & $0.047^{\mathrm{a}}$ & 0.042 \\
Healthcare costs covered & $0.027^{\mathrm{a}}$ & 0.021 \\
Spiritual advisor visits & $0.045^{\mathrm{a}}$ & 0.040 \\
Funeral arrangements in order & $0.027^{\mathrm{a}}$ & 0.021 \\
\hline
\end{tabular}

OODD: quality of dying and death.

aMann-Whitney test.

\section{General review by participants}

For the "QODD of decedent's last days," the rating of the relatives of the intensive care group was lower than that of the hospice group ( 4.3 vs. $6.9, \mathrm{P}=0.126$ ), although this result was not significant.

\section{Subgroup Comparison on the QODD in the Intensive Care Group}

In the intensive care group, some respondents rated a higher total QODD score than others. The ratings for the following items were significantly higher in the rater group with higher QODD scores than those in the latter group: "untroubled about strain on loved ones," "healthcare costs covered," "spiritual advisor visits," and "funeral arrangements" (Table 4). In this group, two patients were transferred out of the ICU for end-oflife care in their last days and died in a general ward death bed. The ratings of the QODD from the relatives of these two patients were higher than those of the relatives whose loved ones died in the ICU (median, 64.5 vs. 45.0, $\mathrm{P}=0.095$ ).

\section{DISCUSSION}

Settings of ICUs are not conducive to the provision of palliative care. However, it has become an important task for medical professionals in ICUs to provide an appropriate level of palliative care in accordance with the diverse medical conditions of patients [2,11].

The goal of palliative care is to improve quality of life for both the patient and the family by focusing on providing relief from the symptoms and stress of a serious illness [12]. According to this definition, palliative care should not only provide continuous medical care until death, but should also provide various evidence-based interventions aimed at relieving patients' pain and their relatives' suffering [11]. Therefore, the experience of patients and their relatives is an important factor in assessing the quality of palliative care in ICUs.

However, it can be argued that a better way of assessing the quality of palliative care is to directly ask patients with terminal illnesses and their caregivers questions about their experiences [10]. Evaluating patients and their family members' experience could identify unsatisfactory healthcare services and provide opportunities to improve the quality of hospital care. Understanding the current level of quality of care and identifying performance goals must precede improving the quality of care $[11,13]$.

Previous studies have identified and presented the key areas of the QODD through various research methods, including questionnaire surveys, group interviews with patients and relatives, and expert opinions and intervention studies [1419]. Patrick et al. [3] presented the six areas of the QODD: "symptoms and personal care," "preparation of death," "moment of death," "family," "treatment of preferences," and "whole person concerns." The above measuring tools broadly encompass the two key aspects of the QODD: "appropriateness of scientific and medical care" and "appropriateness of personal and cultural aspects of care." This approach supports the fact that many studies assessing the QODD of critically ill patients in the ICUs use such domains and items [20,21].

This study is based on comparative assessments of the QODD questionnaire by family members whose loved one died either in the ICU or in hospice. There a couple potential issues related to this methodology. First, compared to the hospice, the ICU has very different functions and purposes. Therefore, it could be that the characteristics of the patients from these two groups are not identical. However, there are two commonalities between the two groups. First, medical professionals were able to predict patients' incurability and death. Although the underlying causes were different, all the decedents in the two groups were irreversibly incurable regarding their medical condition. In this study, advance care planning including DNR orders had been given to critically ill patients in the intensive care group as well as to terminal cancer patients in hospice care. Second, patients from both groups needed palliative care and end-of-life care during their last days. The hospice patients received standardized palliative care and endof-life care during their last days. However, the design to allow the omission of essential palliative care on the predetermined grouping even in the same ICU would lead to critical ethical conflict. Therefore, as demonstrated by previous studies, the hospice group can help us to identify unmet needs of the pa- 
tients from the ICU $[22,23]$.

A second issue could be that the experience and perception of the deceased are not necessarily the same as their relatives' [3]. The main participants of this study are the close relatives who cared for the deceased. It is quite possible that the relatives may not be able to accurately assess symptoms, care experience, and the perceptions of the patients [3]. However, it is important to acknowledge that the assessments from the relatives based on their experience of caring would closely reflect the actual perceptions of the quality of care that healthcare providers may not identify $[3,17]$.

The QODD total score and QODD by domains and items in the ICU group are lower than those of the hospice group, which was the natural expectation in this study. However, there are a few findings from this study. First, the survey participation rate from the relatives of the intensive care group was lower $(n=16,50.0 \%)$ than that of the hospice group. The main reason given for the low participation was to not "bring back the memories." The relatives of the hospice group would receive emotional support from the hospice during their grieving process. Conversely, the relatives of patients in intensive care do not generally receive such emotional support, even though death can occur rather suddenly. There was no way of knowing how the nonparticipating relatives would have assessed the QODD, but it is reasonable to assume that the high decline rate and the reason for its declining reflect an inappropriate level of care in the ICUs.

Second, this study found that for both groups, "opportunity to avoid or choose life-sustaining treatments" was important and was correlated with the QODD. In Korean culture, terminally ill patients had difficulty determining even their own life-sustaining treatments regarding their previous advance directives by February 2018. This may hinder the essential advance care planning process for improving quality of dying and death. Many physicians feel uncomfortable in beginning and leading conversations regarding end-of-life care for multiple reasons, including personal attitudes [24,25]. These imply that education for healthcare professionals should be provided to enhance clinicians' performance in caring for dying patients in the ICUs. Recent guidelines also support that medical professionals should have knowledge and competence for end-of-life care [2,26,27].

Last, this study found that relatives needed enough time to accept and prepare for their loved one's death once the treatment to prolong the patient's life was determined to be futile. In the intensive care group, respondents with a higher QODD experience were highly satisfied with questionnaire items such as "spiritual advisor visits," "funeral arrangements," "goodbyes said," and "time with children." Such items are associated with better end-of-life care. For this, shared decision making, including advance care planning, should be encouraged early in ICUs. Many studies found that good communication with medical professionals was a major factor affecting satisfaction of the relatives [28,29]. In ICUs, good communication and shared decision making among stakeholders may provide dying patients with better end-of-life care to improve their QODD in their last days $[27,30,31]$.

This study designed a retrospective survey of the QODD to identify unmet needs for the care of dying patients in ICUs. By assessing the intensive care group relatives' perceptions of the QODD, this study suggests, as have previous studies, that there are unmet needs for the care of dying patients in the ICU. A few limitations of this study should be noted. The participants of the study were possibly biased, as the participants were from a single referral hospital. Further, since the participants were a small group, selection bias could not be avoided. The assessment tool, i.e., the QODD, was developed for those in the United States. Therefore, social and cultural differences should be acknowledged and a more measured interpretation of the results from this study is needed.

In conclusion, this study found that in this tertiary referral hospital's ICU, there are unmet needs for the care of dying patients and for the quality of dying and death. This result suggests that shared decision making for advance care planning should be encouraged and that education on caring for dying patients should be provided to healthcare professionals to improve the quality of dying and death in Korean ICUs.

\section{CONFLICT OF INTEREST}

No potential conflict of interest relevant to this article was reported.

\section{ACKNOWLEDGMENTS}

This study was completed as a policy research project (2017) with the financial support of the Korea National Institute for Bioethics Policy (KoNIBP).

We are indebted to Yeon-sil Choi and Young Sim Choi for the data collection of this article. Also we are grateful to Hospice Care Team of Chungnam National University Hospital for their hard work. 


\section{ORCID}

Yanghwan Choi

Myoungrin Park

Da Hyun Kang

Jooseon Lee

Jae Young Moon

Heejoon Ahn https://orcid.org/0000-0002-9616-2252

https://orcid.org/0000-0002-9080-6256

https://orcid.org/0000-0002-3495-0931

https://orcid.org/0000-0001-8839-3264

https://orcid.org/0000-0001-8724-6289

https://orcid.org/0000-0002-7075-7565

\section{AUTHOR CONTRIBUTIONS}

Conceptualization: MP, JYM. Data curation: YC, JL. Formal analysis: YC, JYM. Funding acquisition: MP. Methodology: YC, MP, JYM. Project administration: DHK, JYM. Visualization: DHK. Writing - original draft: YC, JYM. Writing - review \& editing; JYM, HA.

\section{SUPPLEMENTARY MATERIALS}

The online-only supplement data are available with this article online: https://doi.org/10.4266/acc.2018.00374.

\section{REFERENCES}

1. Statistics Korea. Report of 2017 birth and death rate. Daejeon; Statistics Korea; 2018.

2. Truog RD, Campbell ML, Curtis JR, Haas CE, Luce JM, Rubenfeld GD, et al. Recommendations for end-of-life care in the intensive care unit: a consensus statement by the American College [corrected] of Critical Care Medicine. Crit Care Med 2008;36:953-63.

3. Patrick DL, Engelberg RA, Curtis JR. Evaluating the quality of dying and death. J Pain Symptom Manage 2001;22:717-26.

4. Curtis JR, Patrick DL, Engelberg RA, Norris K, Asp C, Byock I. A measure of the quality of dying and death; initial validation using after-death interviews with family members. J Pain Symptom Manage 2002;24:17-31.

5. Heckel M, Bussmann S, Stiel S, Weber M, Ostgathe C. Validation of the German Version of the Quality of Dying and Death Questionnaire for Informal Caregivers (QODD-D-Ang). J Pain Symptom Manage 2015;50:402-13.

6. Pérez-Cruz PE, Padilla Pérez O, Bonati P, Thomsen Parisi O, Tupper Satt L, Gonzalez Otaiza M, et al. Validation of the Spanish version of the Quality of Dying and Death Questionnaire (QODD-ESP) in a home-based cancer palliative care program and development of the QODD-ESP-12. J Pain Symptom Manage 2017;53:1042-9.
7. Cho JY, Lee J, Lee SM, Park JH, Kim J, Kim Y, et al. Transcultural adaptation and validation of quality of dying and death questionnaire in medical intensive care units in South Korea. Acute Crit Care 2018;33:95-101.

8. Barr J, Fraser GL, Puntillo K, Ely EW, Gélinas C, Dasta JF, et al. Clinical practice guidelines for the management of pain, agitation, and delirium in adult patients in the intensive care unit. Crit Care Med 2013;41:263-306.

9. National Cancer Center. Cancer pain management guideline. Goyang: National Cancer Center; 2015.

10. Downey L, Curtis JR, Lafferty WE, Herting JR, Engelberg RA. The Quality of Dying and Death Questionnaire (QODD): empirical domains and theoretical perspectives. J Pain Symptom Manage 2010;39:9-22.

11. Mularski RA. Defining and measuring quality palliative and end-of-life care in the intensive care unit. Crit Care Med 2006; 34(11 Suppl):S309-16.

12. Center to Advance Palliative Care. About palliative care. New York: Center to Advance Palliative Care; 2018.

13. Blumenthal D. Part 1: quality of care: what is it? N Engl J Med 1996;335:891-4

14. Steinhauser KE, Christakis NA, Clipp EC, McNeilly M, Grambow S, Parker J, et al. Preparing for the end of life: preferences of patients, families, physicians, and other care providers. J Pain Symptom Manage 2001;22:727-37.

15. Steinhauser KE, Christakis NA, Clipp EC, McNeilly M, McIntyre L, Tulsky JA. Factors considered important at the end of life by patients, family, physicians, and other care providers. JAMA 2000;284:2476-82.

16. Singer PA, Martin DK, Kelner M. Quality end-of-life care: patients' perspectives. JAMA 1999;281:163-8.

17. Lynn J, Teno JM, Phillips RS, Wu AW, Desbiens N, Harrold J, et al. Perceptions by family members of the dying experience of older and seriously ill patients; SUPPORT investigators. Study to understand prognoses and preferences for outcomes and risks of treatments. Ann Intern Med 1997;126:97-106.

18. Clarke EB, Curtis JR, Luce JM, Levy M, Danis M, Nelson J, et al. Quality indicators for end-of-life care in the intensive care unit. Crit Care Med 2003;31:2255-62.

19. American Academy of Hospice and Palliative Medicine; Center to Advance Palliative Care; Hospice and Palliative Nurses Association; Last Acts Partnership; National Hospice and Palliative Care Organization. National Consensus Project for Quality Palliative Care: Clinical Practice Guidelines for quality palliative care, executive summary. J Palliat Med 2004;7:611-27.

20. Gerritsen RT, Koopmans M, Hofhuis JG, Curtis JR, Jensen HI, Zijlstra JG, et al. Comparing quality of dying and death per- 
ceived by family members and nurses for patients dying in US and Dutch ICUs. Chest 2017;151:298-307.

21. Levy CR, Ely EW, Payne K, Engelberg RA, Patrick DL, Curtis JR. Quality of dying and death in two medical ICUs: perceptions of family and clinicians. Chest 2005;127:1775-83.

22. Teno JM, Clarridge BR, Casey V, Welch LC, Wetle T, Shield R, et al. Family perspectives on end-of-life care at the last place of care. JAMA 2004;291:88-93.

23. Kinoshita H, Maeda I, Morita T, Miyashita M, Yamagishi A, Shirahige $Y$, et al. Place of death and the differences in patient quality of death and dying and caregiver burden. J Clin Oncol 2015;33:357-63.

24. Phua J, Joynt GM, Nishimura M, Deng Y, Myatra SN, Chan YH, et al. Withholding and withdrawal of life-sustaining treatments in intensive care units in Asia. JAMA Intern Med 2015;175:36371.

25. Park SY, Phua J, Nishimura M, Deng Y, Kang Y, Tada K, et al. End-of-life care in ICUs in East Asia: a comparison among China, Korea, and Japan. Crit Care Med 2018;46:1114-24.

26. Lanken PN, Terry PB, Delisser HM, Fahy BF, Hansen-Flaschen J, Heffner JE, et al. An official American Thoracic Society clinical policy statement: palliative care for patients with respiratory diseases and critical illnesses. Am J Respir Crit Care Med
2008;177:912-27.

27. Sprung CL, Truog RD, Curtis JR, Joynt GM, Baras M, Michalsen A, et al. Seeking worldwide professional consensus on the principles of end-of-life care for the critically ill: the consensus for Worldwide End-of-Life Practice for Patients in Intensive Care Units (WELPICUS) study. Am J Respir Crit Care Med 2014;190:855-66.

28. Gerritsen RT, Hofhuis JG, Koopmans M, van der Woude M, Bormans L, Hovingh A, et al. Perception by family members and ICU staff of the quality of dying and death in the ICU: a prospective multicenter study in The Netherlands. Chest 2013; 143:357-63.

29. Piers RD, Azoulay E, Ricou B, Dekeyser Ganz F, Decruyenaere J, Max A, et al. Perceptions of appropriateness of care among European and Israeli intensive care unit nurses and physicians. JAMA 2011;306:2694-703.

30. Cook D, Rocker G. Dying with dignity in the intensive care unit. N Engl J Med 2014;370:2506-14.

31. Kon AA, Davidson JE, Morrison W, Danis M, White DB; American College of Critical Care Medicine, et al. Shared decision making in ICUs: an American College of Critical Care Medicine and American Thoracic Society Policy statement. Crit Care Med 2016;44:188-201. 
Supplementary Table 1. Medical condition and LST of the decedents in the ICU

\begin{tabular}{|c|c|c|c|c|}
\hline No. & Age $(y r) / s e x$ & Diagnosis at ICU admission & MOF \& medical condition & $\mathrm{LST}^{\mathrm{a}}$ in last days \\
\hline 1 & $80 / F$ & Sepsis, pulmonary edema & SCMP, AKI, ARF, DIC & MV, CRRT, vasopressor \\
\hline 2 & $72 / \mathrm{M}$ & Lung cancer, pneumonitis & AKI, ARF, DIC & MV, ECMO, CRRT vasopressor \\
\hline 3 & $85 / \mathrm{M}$ & Lymphoma, septic shock & AKI, DIC, ARF & $\begin{array}{l}\text { CRRT and MV were weaned successfully, } \\
\text { but medical condition deteriorated to death. }\end{array}$ \\
\hline 4 & $72 / \mathrm{M}$ & Lung cancer, pneumonia & ARF & MV \\
\hline 5 & $83 / \mathrm{F}$ & NSTEMI, AHF & $A H F, A R F, A K I$ & MV, ECMO, vasopressor \\
\hline 6 & $81 / \mathrm{M}$ & Candidemia, COPD, ARDS, Cor pulmonale & AKI, ARF & MV, CRRT, vasopressor \\
\hline 7 & $69 / \mathrm{M}$ & Acute cerebral infarction, adrenal insufficiency & ARF, refractory shock & MV, vasopressor \\
\hline 8 & $85 / \mathrm{M}$ & Septic shock, ARDS & ARF & MV, vasopressor \\
\hline 9 & $71 / F$ & Pneumonia, RA, sepsis & ARF, immunosuppression & MV, vasopressor \\
\hline 10 & $80 / \mathrm{M}$ & COPD, pneumonia & ARF, AKI & CRRT, vasopressor, withdrawing/withholding of MV \\
\hline 11 & $86 / \mathrm{M}$ & $A H F, C K D$, femur fracture & ARF, CRF & MV, CRRT, vasopressor \\
\hline 12 & $87 / \mathrm{M}$ & COPD, pneumonia, pulmonary thromboembolism & ARF & MV \\
\hline 13 & $75 / \mathrm{M}$ & Multiple myeloma, septic shock, candidemia & Refractory shock & MV, vasopressor \\
\hline 14 & $84 / F$ & Whole bowel necrosis, septic shock, ischemic colitis & AKI, ARF & $\begin{array}{l}\text { MV, CRRT, vasopressor withdrawing/withholding of } \\
\text { MV \& CRRT }\end{array}$ \\
\hline 15 & $83 / \mathrm{M}$ & COPD, cor pulmonale, pneumonia, post-CPR & ARF, RV failure & MV, vasopressor \\
\hline 16 & $87 / \mathrm{M}$ & ARDS due to trauma & AKI, ARF & MV, vasopressor \\
\hline
\end{tabular}

LST: life-sustaining treatment; ICU: intensive care unit; MOF: multiple organ failure; F: female; M: male; SCMP: stress-induced cardiomyopathy; AKI: acute kidney injury; ARF: acute respiratory failure; DIC: disseminated intravascular coagulation; MV: mechanical ventilation; CRRT: continuous renal replacement therapy; ECMO: extracorporeal membrane oxygenation; NSTEMI; non- ST-elevation myocardial infarction; AHF: acute heart failure; COPD: chronic obstructive pulmonary disease; ARDS: acute respiratory distress syndrome; RA: rheumatoid arthritis; CKD: chronic kidney disease; CRF: chronic renal failure; CPR: cardiopulmonary resuscitation.

a"LST" is defined as the following: medical procedures that would only prolong the process of dying or sustain a condition of permanent unconsciousness. A patient who is receiving LST will die soon, whether or not treatment is administered administered [1].

\section{REFERENCE}

1. Legal Information Institute. Life-sustaining treatment [Internet]. New York (NY): Legal Information Institute, Cornell Law School [cited 2019 Jun 20]. Available from: https://www.law.cornell.edu/wex/life-sustaining_treatment. 